\title{
Economic analysis of the impact of exchange rate changes on the production costs of wheat crop in Egypt
}

\author{
Osman B. H. M. , Ismail O. A., Ahmed A. I. M. \\ Agricultural Economics Department, Faculty of Agriculture, Al-Azhar University, Assuit, Egypt
}

\begin{abstract}
The decline in the exchange rate of the Egyptian pound and the decline in domestic production of wheat crop and its inadequacy for domestic consumption of wheat, and as the population widens the food gap of wheat and increases its cost. The study was based on descriptive and quantitative analysis methods to analyze the data on the subject of the study through the use of certain mathematical and statistical methods, The increase in costs was shown at an annual rate of increase influenced by changes in the exchange rate of the Egyptian pound estimated at 584.5 pounds per acre, at an annual rate of change of about $18.98 \%$ of the average, while the moral its of its impact on the total costs did not prove to be fixed prices because the stabilization of prices disappeared the impact of exchange rate changes. Recommendations suggested by farmers were as follows:
\end{abstract}

- The need to provide production supplies at appropriate prices to encourage farmers and reduce dependence on abroad to provide foreign currency.

- $\quad$ Search for alternatives to production supplies and encourage the industry of the national imported alternative.

Keywords: exchange rate, total costs, farm prices.

* Corresponding author: Osman B. H. M.,

E-mail address: balegh2020@ azhar.edu.eg 


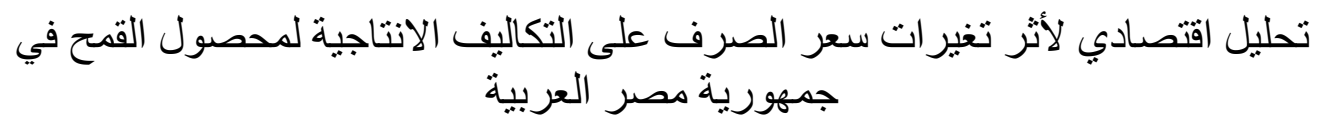

\author{
بليغ حمدي محمد عثمان ، عثمان علي إسماعيل ، أحمد ابر اهيم محمد أحمد \\ قسم الإقتصاد الزر اعي ، كلية الزر اعة ، جامعة الأزهر - فرع أسبوط ، جمهورية مصر العربية
}

بعتبر سعر صرف الجنيه المصري أحد المتغير ات الهامة التي تهتم بها الدول وخاصة الدولة المصرية نتيجة لزيادة طلبها على العملات الأجنبية

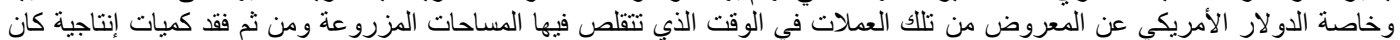
يمكن انتاجها كل ذلك أدت إلى تدهور مستمر لقيمة الجنية المصري الإني أمام العملات الأجنبية المختلفة.

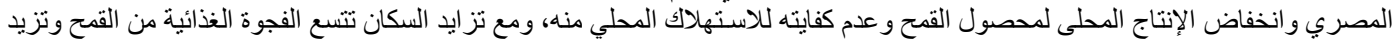

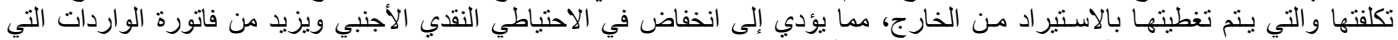

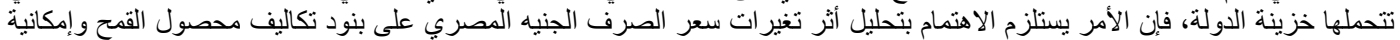

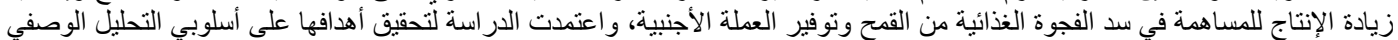

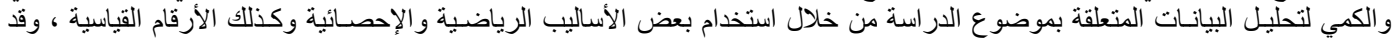

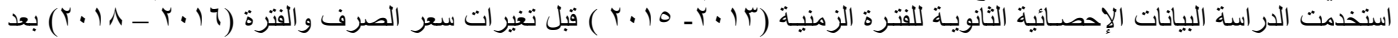

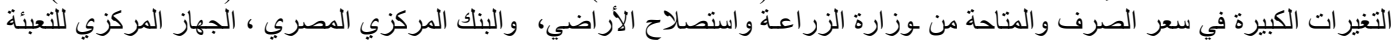

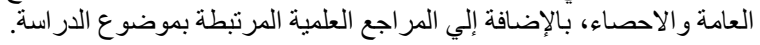




\section{الهُف من البحث}

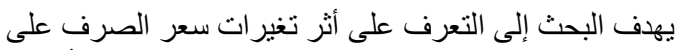

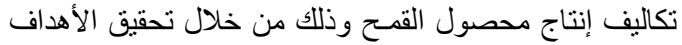

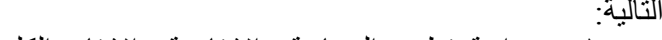
أ: الإسة تطور المساحة والإنتاجية و الإنتاج الكلي

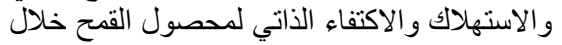

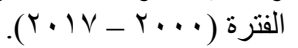
Y. . دراسة كل من التكاليف الكلية والاسعار المزرعية وصافي العائد الفداني لمحصول الفيف القمح خلال الفترة (r.IV-r...)

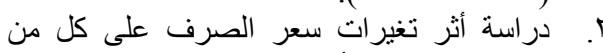

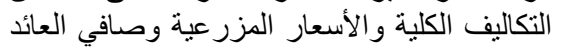

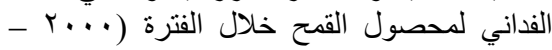
(

ع. تحليل بنود التكاليف الإنتاجية لمحصول القمح قبل فيل

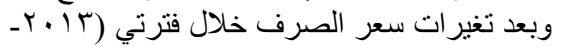

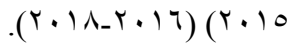

\section{الطريقة البحثية ومصادر جمع البيانات}

اعتمد البحث لتحقيث أهدافه على أسلوبي التحليل الوصفي

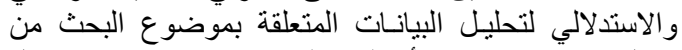
خلال استخدام بعض الأسـاليب الرياضـية والإحصـائية مثنل الإنل

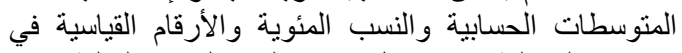

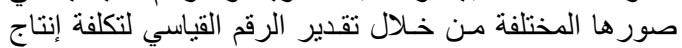

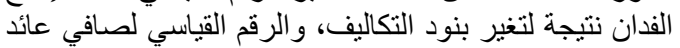

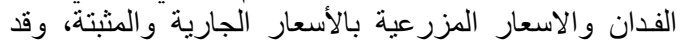

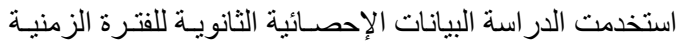

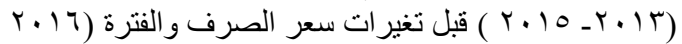

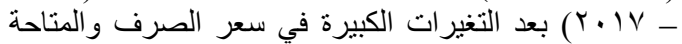

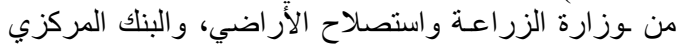

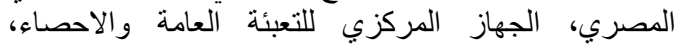

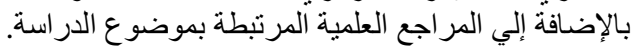

\section{النتائج ومناقشتها}

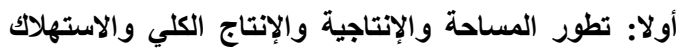

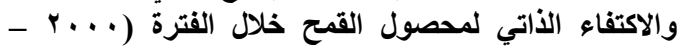

(r. IV

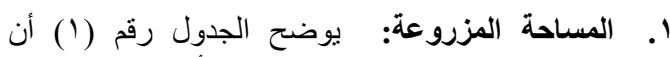

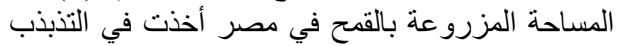

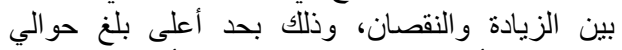

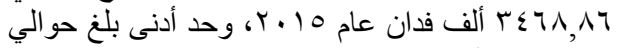

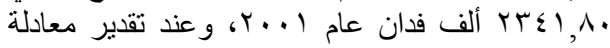
الاتجاه الزمني العام للمساحة المزرو الفة المة، توضح المعادلة
مقدمة - - مقة

يعتبر سعر صرف الجنيه المصري أحد المتغيرات الهامة التي لهاي

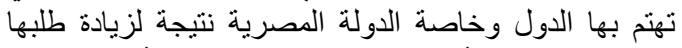

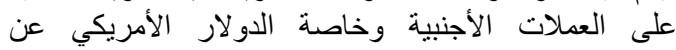

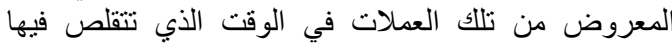

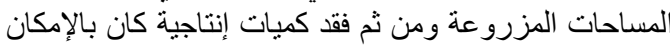

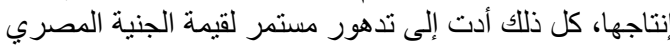

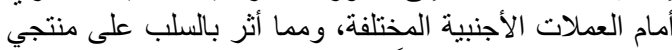

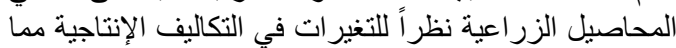

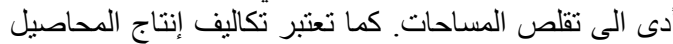

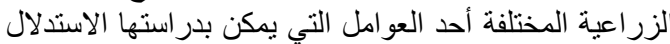

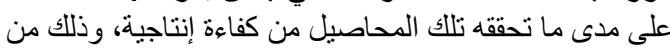

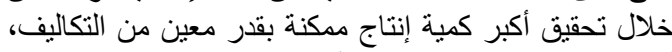

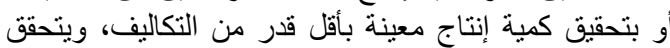

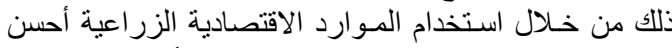

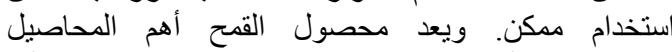

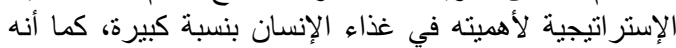

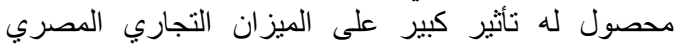

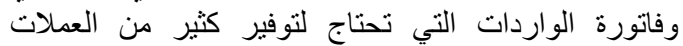

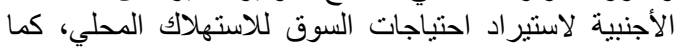

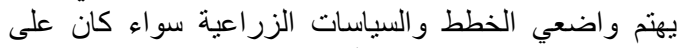

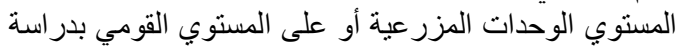

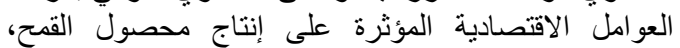

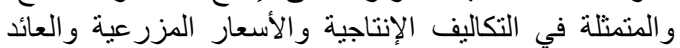

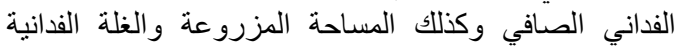

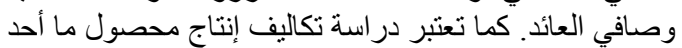

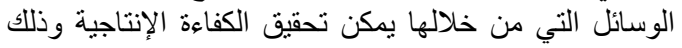

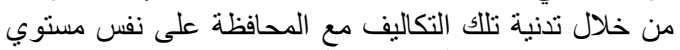

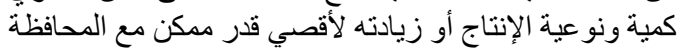
على التكاليف عند مستوي معين.

\section{مشكلة البحث}

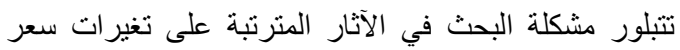

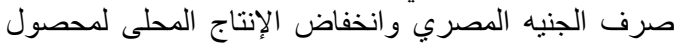

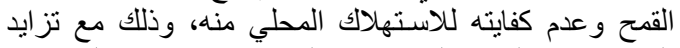

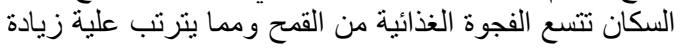

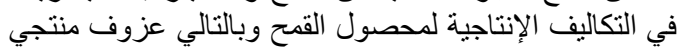

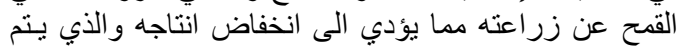

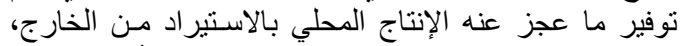

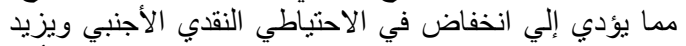

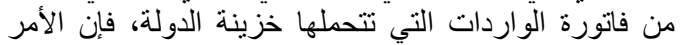

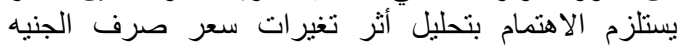

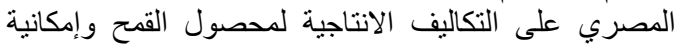
زيادة الإنتاج للمساهمة في سد الفجوة الغذائية من القمح ونوفير والثير العملة الأجنبية. 


$$
\text { المستخدم عند مستوى معنوية (0. ( • •). }
$$

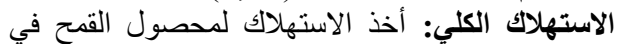

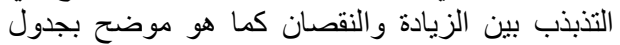

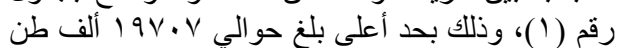

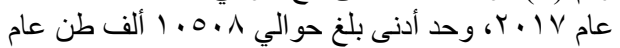

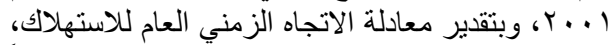

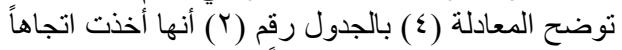

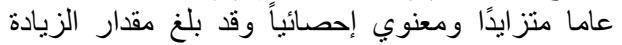

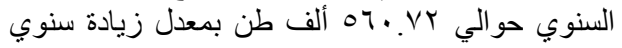

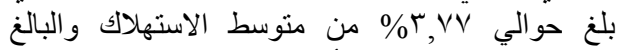

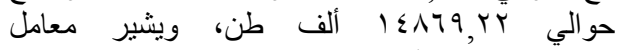

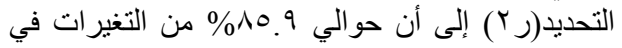

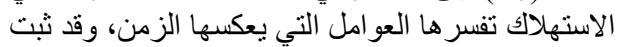

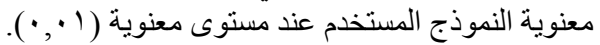

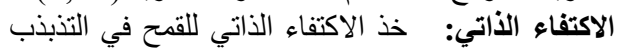

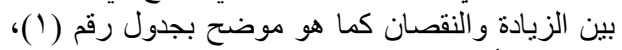

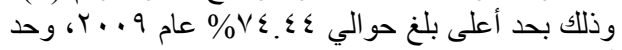

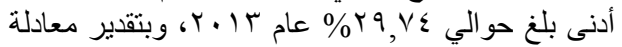

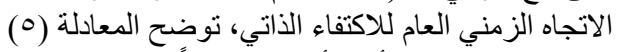

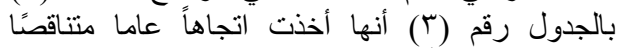

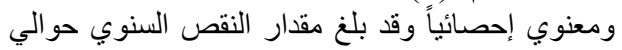

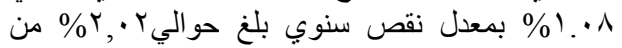

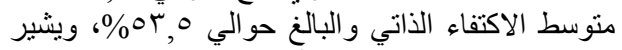

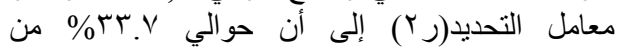
التغير ات في الاكتفاء الذاتي تفسر ها العان العو امل التي يعكسها

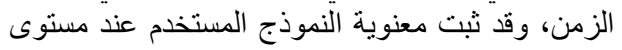

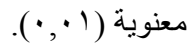

(1) بالجدول رقق (Y) أنها أخذت انجاهاً عاما متزايدًا

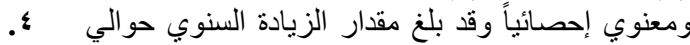

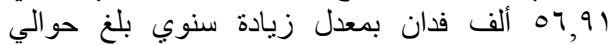

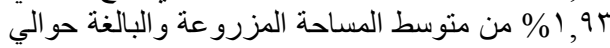

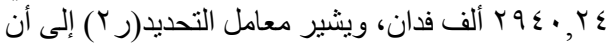

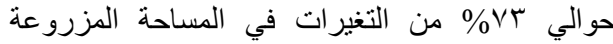

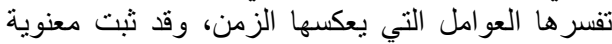

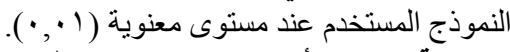

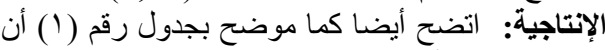

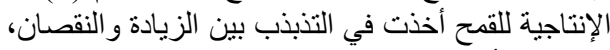

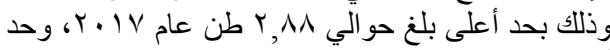

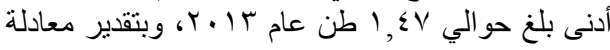

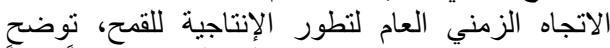

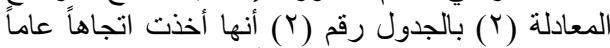

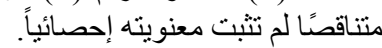

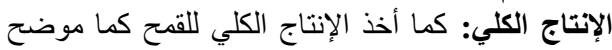

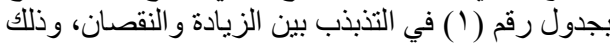

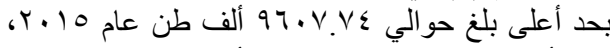

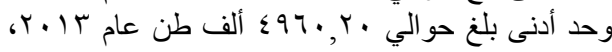

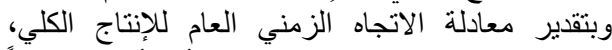

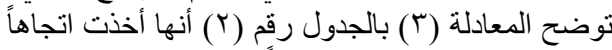

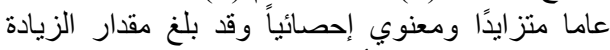

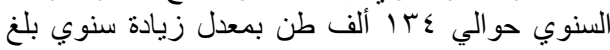

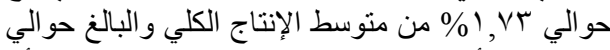

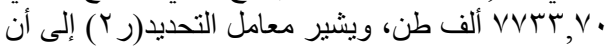

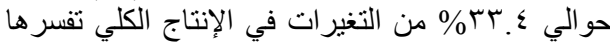

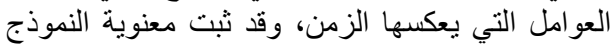

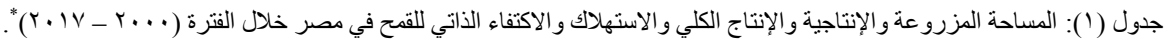

\begin{tabular}{|c|c|c|c|c|c|}
\hline الاكتفاء الذاتي & الاستهلاك & الإنتاج الكلي & الإنتاجية & المساحة المزروعة & السنة \\
\hline 71.79 & $1,7 \leqslant 1, \ldots$ & $707 \varepsilon .0$ & T.77 & $Y \leqslant T Y . Y V$ & r... \\
\hline $09.0 Y$ & $1.0 .1 . \cdots$ & $7 Y 0 \leqslant .01$ & Y.TV & $T r \leqslant 1 . \wedge$. & $r \ldots 1$ \\
\hline or.r & ITEYY.. & TTY & T.V. & $r \leqslant 0 . . \leqslant r$ & Y... \\
\hline Tr. $\leqslant 7$ & $1.901 . \cdots$ & $71 \leq \leqslant .79$ & T.VT & Y0.7.11 & $r \ldots r$ \\
\hline 71.1. & $\| \vee \leqslant \varepsilon \ldots$ & VIVV.AT & T.Vo & $r 7.0 \leqslant 1 \lambda$ & $r \ldots \varepsilon$ \\
\hline 71.17 & |rr|... & N)ะ..97 & T.VT & rq10. ${ }^{9}$ & r..o \\
\hline 0.91 & $1 \leqslant Y \wedge \wedge .$. & AYVE.YT & Y.V. & r.ד.V. & $r \ldots T$ \\
\hline 04.01 & $1 r v q . .$. & VTVA.9Y & Y.VY & YV10.0 & $r \ldots V$ \\
\hline $01 . \varepsilon$. & $1 \leq 0 \leq 7 .$. & $V \leqslant V V .0$ & $Y .07$ & YqY. rA & $r \ldots \lambda$ \\
\hline$V \varepsilon \leqslant \varepsilon$ & $11 \leqslant 0 . .$. & AOYr... & $r . V I$ & $r I \leqslant V . r$ & r.. \\
\hline$\varepsilon \cdot .0 \leqslant$ & $187 \wedge 0 .$. & $V 179 . r$ & r.rq & r... & r.l. \\
\hline$\leqslant \wedge . \wedge$. & IVIOr... & ArV. Or & r.Vo & $r \cdot \leq \wedge .7$. & $r .11$ \\
\hline $00 . \mathrm{VT}$ & lOVAY... & $\Lambda \vee 90 . \leqslant \Lambda$ & Y.VA & $17 . .77$ & Y.IY \\
\hline Yq.V & 177VA... & ह97. Y. & $1 . \leqslant V$ & rTV.AN & $r .1 T$ \\
\hline Or. . T & IVAYO... & $q r \vee q . \wedge$. & r.VT & rrqu... & r.lई \\
\hline 29.11 & $19074 . .$. & $97 . V . V \varepsilon$ & Y.VV & 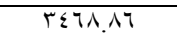 & $r .10$ \\
\hline$\varepsilon \vee .79$ & $19094 . .$. & $9 r \leqslant Y .0 \leqslant$ & Y.V9 & rror.10 & $r .17$ \\
\hline$\varepsilon Y . V T$ & $19 V \cdot V . .$. & $\wedge \leqslant Y) \cdot V$ & 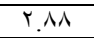 & YQYI.VY & $r . I V$ \\
\hline 0 or.o. & I $\leqslant \wedge 79 . Y Y$ & VVTY.V. & T.Tะ & Yq $\varepsilon . r \varepsilon$ & المتوسط \\
\hline
\end{tabular}


Osman et al. / Archives of Agriculture Sciences Journal 3(1) 81-91, 2020.

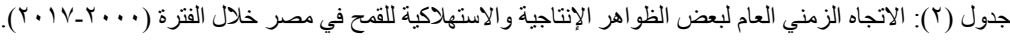

\begin{tabular}{|c|c|c|c|c|c|c|}
\hline معدل التغير (\%) & مقدار التغير & المتوسط & ف & זנ & المعادلة & الظاهرة \\
\hline $1.9 \varepsilon$ & 07,91 & $r 9 \leq ., r \leq$ & $* * \varepsilon r, r q$ & $\cdot,,^{V}+1$ & 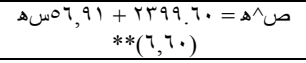 & $\begin{array}{c}\text { (ألف فدان) } \\
\end{array}$ \\
\hline - & - & Y. . & .104 & $\because .9$ & 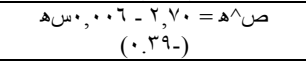 & $\begin{array}{l}\text { (طن/فدان) } \\
\text { (الإنتاجية } \\
\end{array}$ \\
\hline $1, V r$ & $1 \Gamma \varepsilon$ & VVTT.V. & $* \wedge, r q$ & ع & 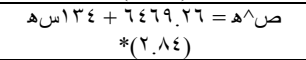 & (ألفِطناج) \\
\hline$r, \mathrm{rv}$ & $07 \cdot, V Y$ & IENT9. KY & $* * q \vee, r$. & $\cdot, 109$ & 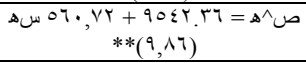 & $\begin{array}{l}\text { ألاستهلاك طن) } \\
\text { (ألف }\end{array}$ \\
\hline T. . T & $1 . \cdot \Lambda_{-}$ & or.o. & $* * \Lambda, 1 r$ & $\cdot, r T V$ & 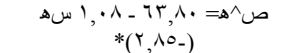 & $\begin{array}{c}\text { الاكتفاء الذاتي } \\
\text { (\%) }\end{array}$ \\
\hline
\end{tabular}

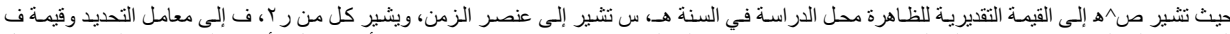

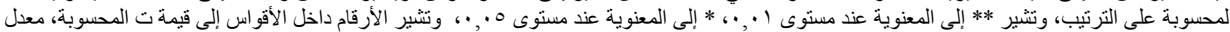

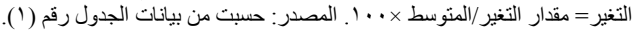

جدول (r): تطور كل من التكاليف الكلية الفدانية والاسعار المزرعية وصافي العائد الفداني بالجنيه لمحصول القمح بالأسعار الجاريـة و المثبتة

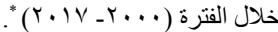

\begin{tabular}{|c|c|c|c|c|c|c|c|}
\hline \multirow{2}{*}{ سعر صرف الجنيه } & \multicolumn{2}{|c|}{ صافي العائد للفدان } & \multicolumn{2}{|c|}{ الأسعار المزرعية } & \multicolumn{2}{|c|}{ التكاليف الكلية للفدان } & \multirow{2}{*}{ السنة } \\
\hline & مثبتة & جارية & مثبتة & جارية & مثبتة & جارية & \\
\hline$r . \leqslant V$ & $9 \cdot V$ & $q \cdot V$ & 790 & 790 & 101. & 101. & r... \\
\hline$r .9 \mathrm{~V}$ & $\Lambda \leqslant \leqslant$ & А१V & $7 N \mathrm{~V}$ & $v \cdot 1$ & $110 \leqslant$ & $10 \mathrm{rr}$ & r... \\
\hline$\{.0$. & $\Lambda \leqslant \leqslant$ & $9 V Y$ & $7 \leqslant 9$ & $\overline{V 1 \Lambda}$ & $T V \leqslant 1$ & 1001 & Y..Y \\
\hline 0.10 & VAT & 1.17 & $7 \ldots$ & V.. & 10rr & $1 \times 10$ & $r \ldots r$ \\
\hline $7 \%$. & $1 . \lambda Y$ & 1777 & $7 V \varepsilon$ & $1 \ldots$ & $10 \leqslant V$ & 19.5 & Y.. \\
\hline $0 . V A$ & 11199 & 1907 & TN & $11 \%$. & ITYY & 1911 & $r \ldots o$ \\
\hline $0 . V T$ & 1.17 & IATr & $\pi r$. & IITV & 11500 & 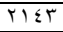 & T.T \\
\hline $0 . T \leqslant$ & ArA & $1 \times 79$ & $07 \pi$ & 1104 & 1 TYA & $r \leqslant \leqslant \leqslant$ & $r \ldots v$ \\
\hline $0 . \varepsilon \Gamma$ & Y..V & 0109 & T.r. & roor & TET & $r 1 \leqslant 0$ & $r \cdots \lambda$ \\
\hline 0.00 & Aт. & Y19. & 701 & $171 \%$ & 17.0 & $r \leqslant 09$ & r.. \\
\hline $0 . T Y$ & $\mathrm{~V} \wedge$ & $19 V \mathrm{~V}$ & TVV & $1 A \mid T$ & $10 \mathrm{~V} \leqslant$ & rin. & r.l. \\
\hline 0.94 & 1.19 & $\lceil\wedge \Lambda \varepsilon$ & 795 & $T r \leqslant V$ & 1094 & $\varepsilon .79$ & $r .11$ \\
\hline 7.7 & $11 \pi v$ & $\leqslant$ rod & $T A V$ & ror. & $10 Y \mathrm{Y}$ & $\leqslant \leqslant r_{0}$ & T.IT \\
\hline $7 . \wedge V$ & $1.1 \mathrm{~V}$ & 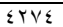 & 717 & rON. & $171 \mathrm{Y}$ & $\varepsilon \wedge \cdot \wedge$ & $r, I r$ \\
\hline 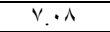 & 991 & 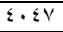 & V.r & TYE. & 177. & OYYI & T.lई \\
\hline$\frac{V . V T}{V T}$ & $94 \leqslant$ & rqई & $7 N T$ & TVOT & $1 \times 91$ & $07 Y V$ & $r .10$ \\
\hline $1 . .14$ & oor & TOVT & 717 & TVVT & $1 \mathrm{ANA}$ & $V .0 \leqslant$ & $r .17$ \\
\hline$I V . V A$ & $7 r 9$ & rAY & 749 & rVר. & IAY. & 1999 & T.IV \\
\hline 7.75 & $9 V 4$ & מדוT & $7 \lambda$. & $1 / 11$ & 1094 & TIYA & المتوسط \\
\hline
\end{tabular}

" تم حساب التكاليف الكلية للفان بالأسعار الثثبتة باستخدام الأرقام القياسية لمستلزمات الإنتاج و الاسعار المزر عبرة المثبتة باستخدام الأرقام القياسية لقيمة الإنتاج

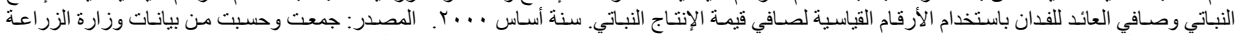

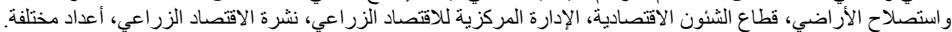

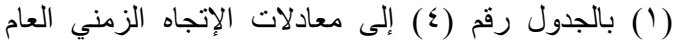

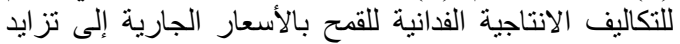

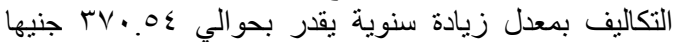

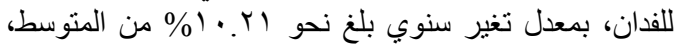

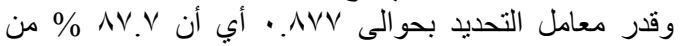

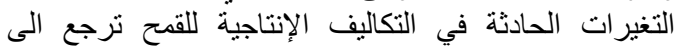
العو امل التي يعكسها عنصر الزمن.

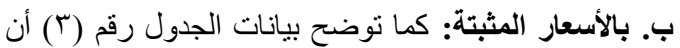

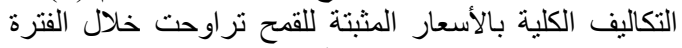

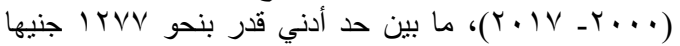

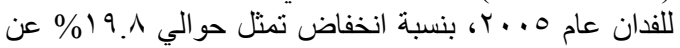

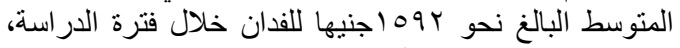

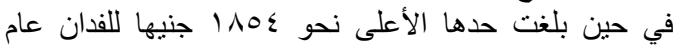

ثانيا: دراسة كل من التكاليف الكلية والاسعار المزرعية

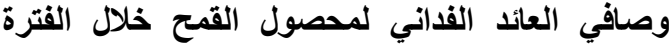

(r. IV - r...)

1. التكاليف الكلية

أ. بالأسعار الجارية: توضح بيانات الجدول رقم (r) أن فئل

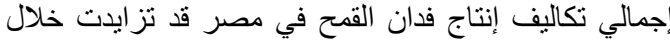

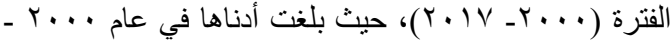

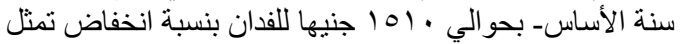

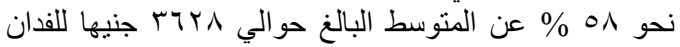

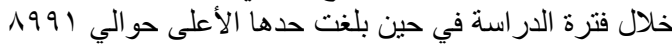

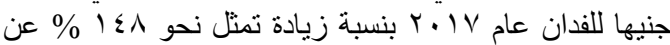
المتوسط السنوي خلال الفترة سالفة الذكر . وتثنير معادلة رقم 
الحادثة في صافي العائد لفدان القمح بالأسعار الجارية ترجع

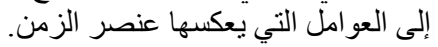

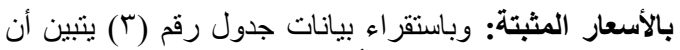

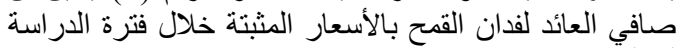

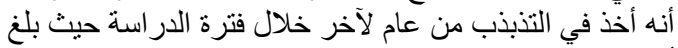

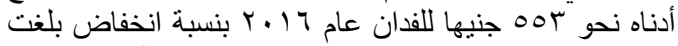

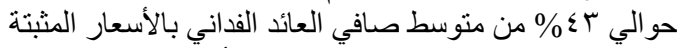
البالغ حوالي

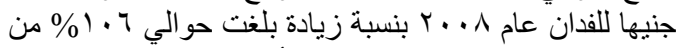

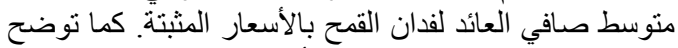

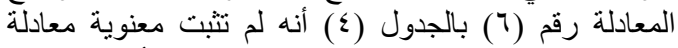
الاتجاه الزمني العام لصافي العائد لفدان القمح بالأسعار المثبتة

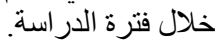

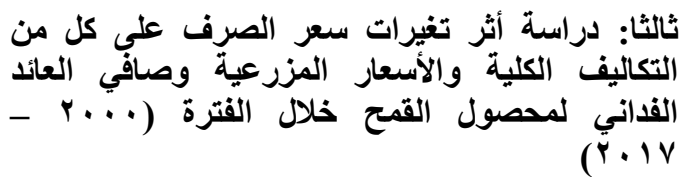

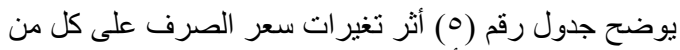

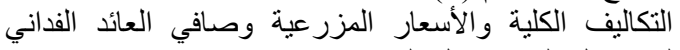

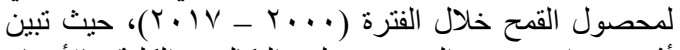
أثر تغيرات سعر الصرف على الفئ التكاليف الكلية بالأسعار

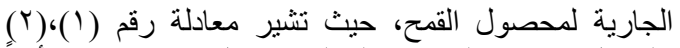

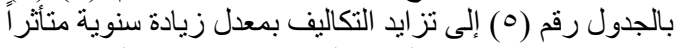

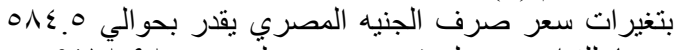

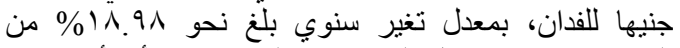

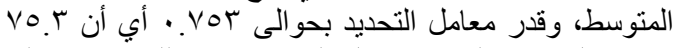

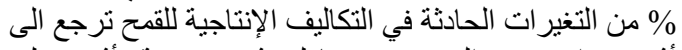

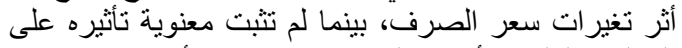

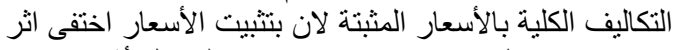

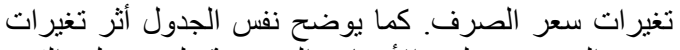

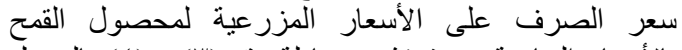

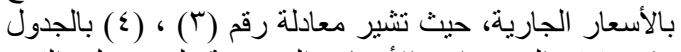

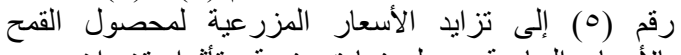

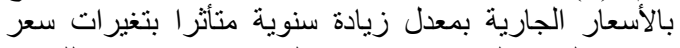

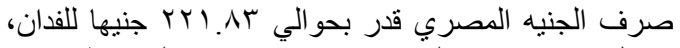

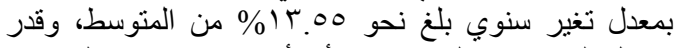

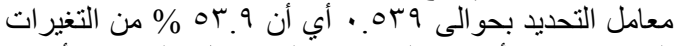

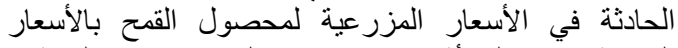

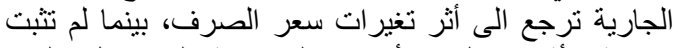

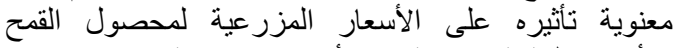

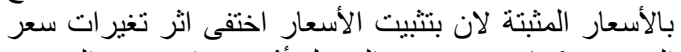

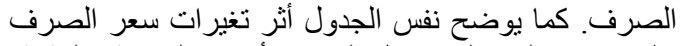

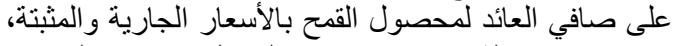

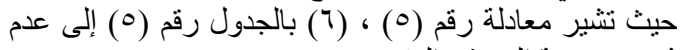
ثبوت معنوية النموذج المقدر.

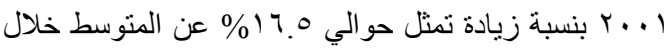

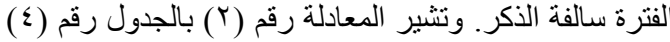

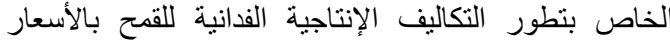
المثبتة إلى إنها لم تثبت معنويتها إحصائيا.

\section{r ب. دراسة الأسعار المزرعية لمحصول القمح}

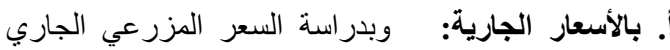

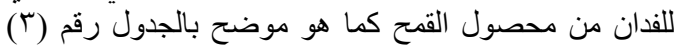

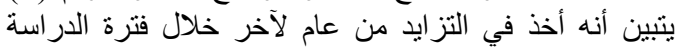

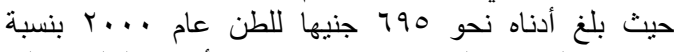

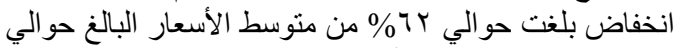

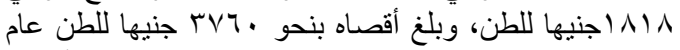

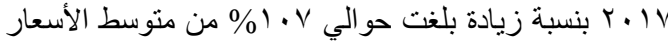

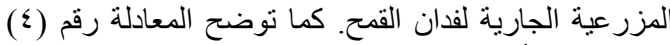

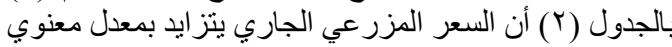

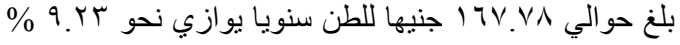

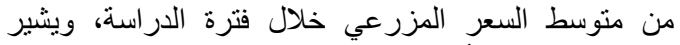

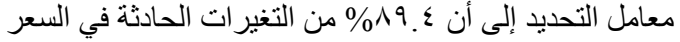

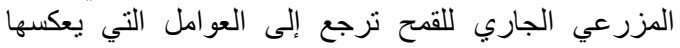

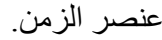

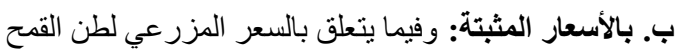

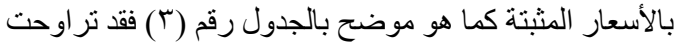

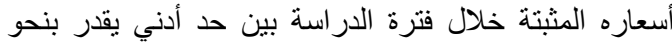

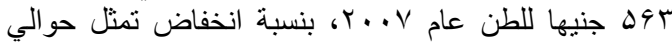

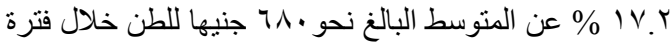

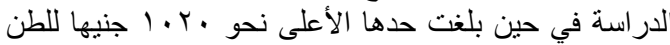

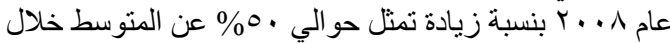

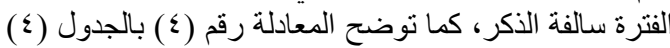

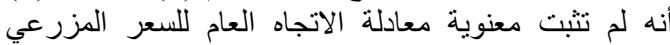
الحقيقي لطن القمح.

\section{r. صافي العائد لفدان القمح}

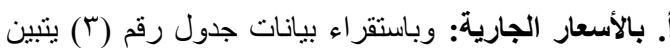

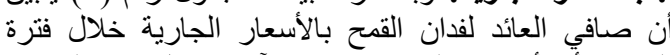

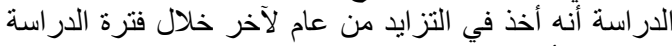

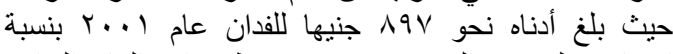

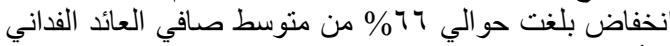

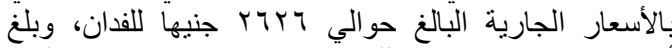

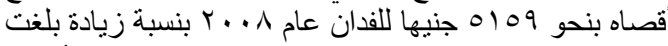

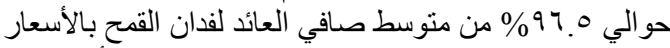

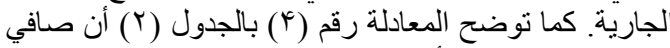

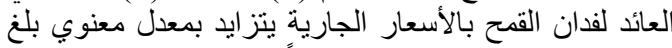

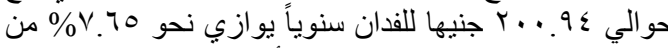

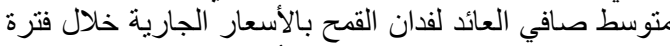

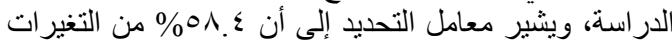


جدول (§): معادلات الاتجاه الزمني العام لنطور كل من التكاليف الكلية الفدانية والاسعار المزرعية وصافي العائد الفداني بالجنيه لمحصول القمح بالأسعار

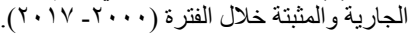

\begin{tabular}{|c|c|c|c|c|c|c|c|c|}
\hline 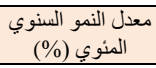 & 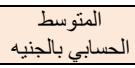 & F & $R^{r}$ & B & A & المعادلة & & البيان \\
\hline $1 \cdot r)$ & rTrA... & $* * \| \leq . Y \wedge$ & $\cdot . \wedge V V$ & $\begin{array}{c}r V \cdot .0 \leq \\
* *(1 \cdot .79)\end{array}$ & $1.1 . .7$ & 1 & جاري) & \multirow{2}{*}{ التكاليف الكلية } \\
\hline$\cdot V \leqslant$ & $1094 . .$. & r. $0 \leqslant$ & $.1 r v$ & $\begin{array}{l}11.20 \\
1.7\end{array}$ & $1 \leqslant \lambda \cdot .7 \lambda$ & r & مثنبة) & \\
\hline Q.rT & $|\wedge| \Lambda_{.}$. & $* * \mid r \leqslant . \wedge r$ & $\cdot . \wedge 9 \varepsilon$ & $\begin{array}{c}17 V . \vee \wedge \\
* *(11.71)\end{array}$ & $r Y \leqslant .19$ & r & (جنية) & \multirow{2}{*}{ المزرعية } \\
\hline . & $7 \wedge . .$. & - & - & $\begin{array}{l}\because \cdot 19- \\
-\because \cdots \leq \\
\end{array}$ & $9 \times 9.90$ & $\varepsilon$ & مثنبة & \\
\hline V.70 & rTY. YA & **rr.01 & $.01 \varepsilon$ & $\begin{array}{l}r \cdots .9 \varepsilon \\
* *(\varepsilon . \vee \varepsilon)\end{array}$ & VIV.rT & 0 & (جنية) & \multirow{2}{*}{ صافي العائد } \\
\hline . & QVर... & $\cdot r \leqslant 4$ & $\because \cdot 10$ & $\begin{array}{l}V_{.} .1 V_{-} \\
. .297_{-}\end{array}$ & $1 . \leqslant 1$ & 7 & مثنبة) & \\
\hline
\end{tabular}

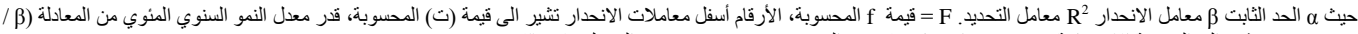

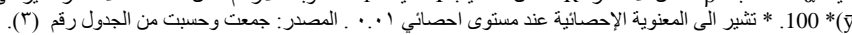

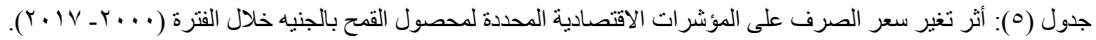

\begin{tabular}{|c|c|c|c|c|c|c|c|c|}
\hline $\begin{array}{c}\text { معدل النمو السنوي (\%) } \\
\text { (الموي }\end{array}$ & الحسابي بالجنيه & $\mathrm{F}$ & $R^{r}$ & B & A & المعادلة & & البيان \\
\hline 11.91 & $r \cdot v \wedge . q$ & $* * \leqslant \wedge . \wedge \uparrow$ & . vor & $\begin{array}{r}010 \\
* *(7.99) \\
\end{array}$ & $r \leq 0 . \wedge-$ & 1 & جارية & \multirow{2}{*}{ التكاليف الكلية } \\
\hline$\cdot V \varepsilon$ & lo9r... & Y.O &. $.1 \mathrm{rV}$ & $\begin{array}{l}Y \\
1.9\end{array}$ & $1 \leqslant \lambda \cdot .7 \lambda$ & r & ثأبتة & \\
\hline $1 . \leqslant \wedge$ & $107 \% . y$ & r.7 & .110 & $\begin{array}{c}r Y Y \\
* *(\xi, r T)\end{array}$ & $1 \leqslant r \lambda$ & $r$ & جارية & \multirow{2}{*}{ المزرعية } \\
\hline . & $7 \wedge \cdot .$. & . & - & $\begin{array}{c}0_{-} \\
-\cdot . T V Y\end{array}$ & $7 \times 9.90$ & $\varepsilon$ & ثابتة & \\
\hline 15.00 & $174 \mathrm{rV} .1$ & $* *|\wedge\rangle T$. & .049 & $\begin{array}{l}180 \\
1.2\end{array}$ & $r \leqslant v . q$ & 0 & أسعار & \multirow{2}{*}{ صافي العائد } \\
\hline . & qvr... & $\cdot r \leqslant 7$ & $\because \cdot 10$ & $\begin{array}{l}r T- \\
1-\end{array}$ & $1 \cdot 1$ & 1 & ثابتة & \\
\hline
\end{tabular}

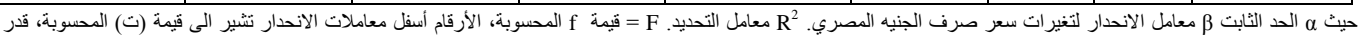

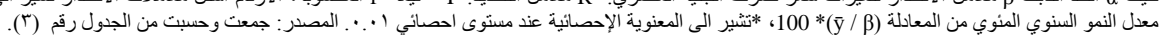

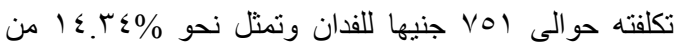

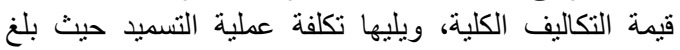

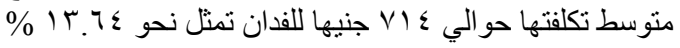

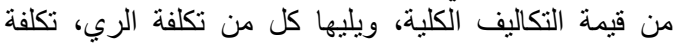

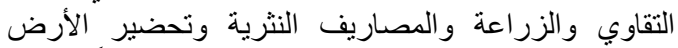

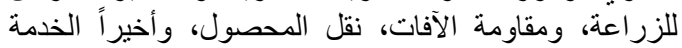

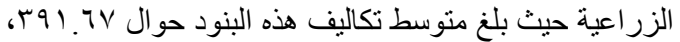

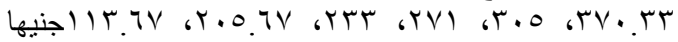

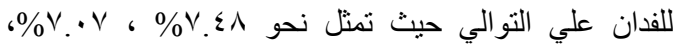

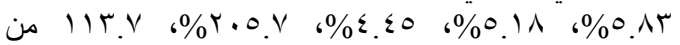

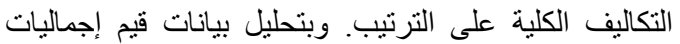

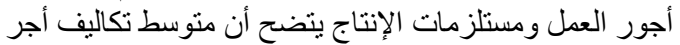

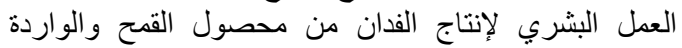

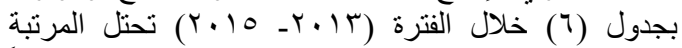

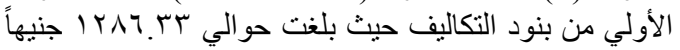

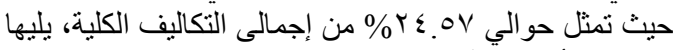

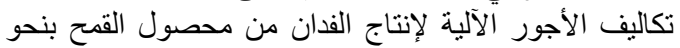

رابعا: تحليل بنود التكاليف الإنتاجية لمحصول القمح فئح

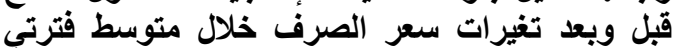

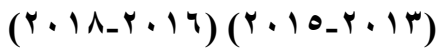

الأهمية النسبية لبنود تكاليف إنتاج فدان القمح خلال الفترة

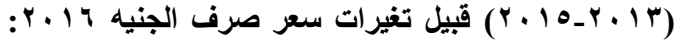

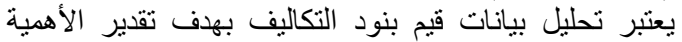

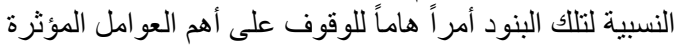

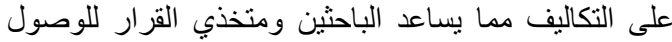

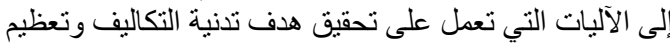

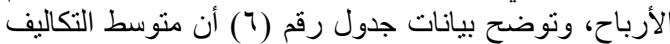

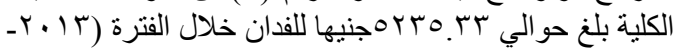

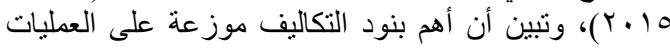

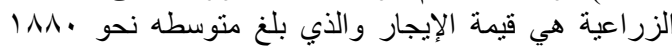

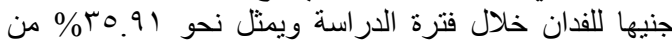

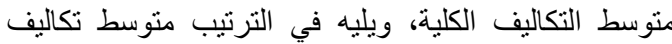
الحصاد والجني الذي احتل المرة الثانية حيث بلغ متوسط 
المتغيرة حسب توزيعها على العمليات الزراعية بلغ حوالي

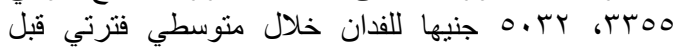

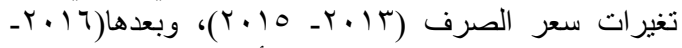

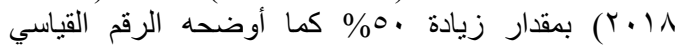

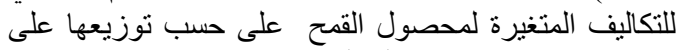

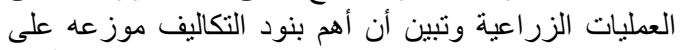

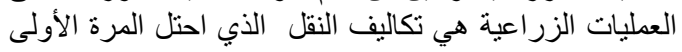

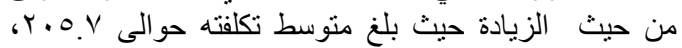

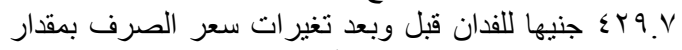

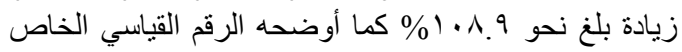

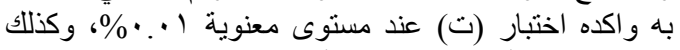

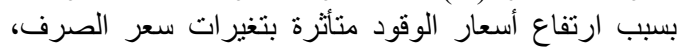

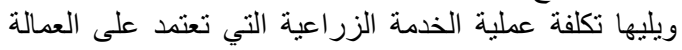

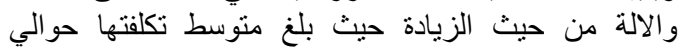

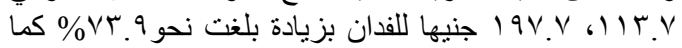

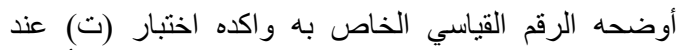

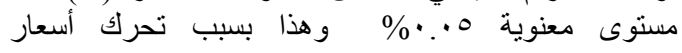

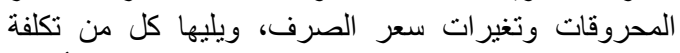

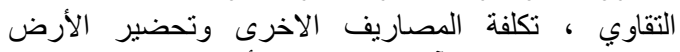

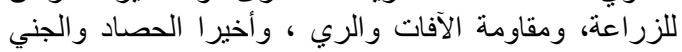

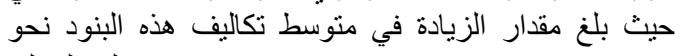

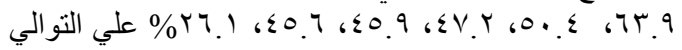

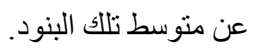

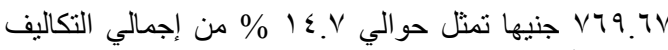

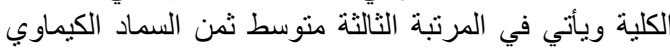

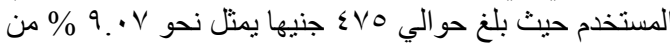

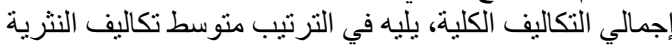

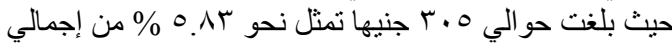

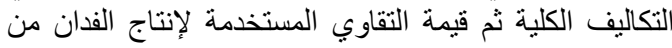

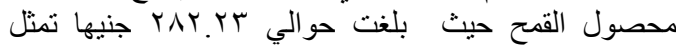

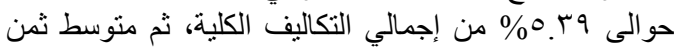

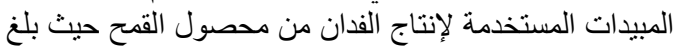

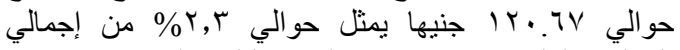

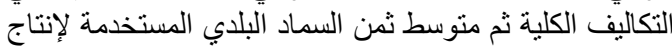

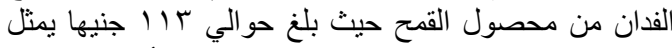

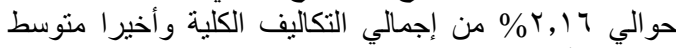

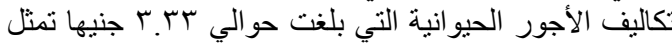
حو الي 7 . . \% من إجمالي التكاليف الكلية خلال فترة الدر اسة.

\section{خامسا: أثر تغير سعر الصرف على بنود التكاليف الإنتاجية لمحصول القمح}

أ. أثر تغير سعر الصرف على بنود تكاليف إنتاج فدان القمح فئح فئحي

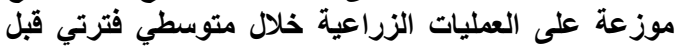

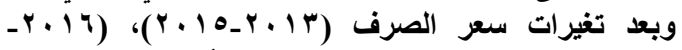

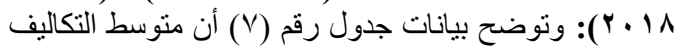

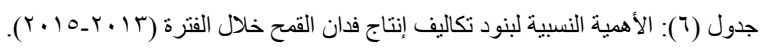

\begin{tabular}{|c|c|c|c|c|c|}
\hline |النسبة المنوية (\%) & متوسط الفترة & التكاليف موز عة الى أجور ومستلزمات انتاج & النسبة المئوية (\%) & متوسط الفترة & التكاليف موزعة على العمليات الزراعية \\
\hline TE.OV & TKAт.rT & أجور عمال & 0.11 & TY... & تحضبر الأرض للزراعة \\
\hline$\because 7$ & r.tr & أجور حيو انات & $\frac{V . V}{V}$ & $r v \cdot r \mu$ & الثقاوي و الزراعة \\
\hline $1 \leqslant . V$. & V79.TV & 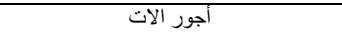 & V. ¿ A & rq1.TV & 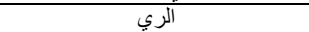 \\
\hline 0.49 & TAT.TT & ثمن الثقاوي & $T$ TT.TE & $V \backslash \leqslant .$. & التسميز \\
\hline 5.17 & $11 \% .$. & ثمن السماد البلذي & Y.IV & $11 \% . T V$ & الخذمة الزراعية \\
\hline $9 . .2$ & $\varepsilon \vee 0_{0} .$. & ثمن السماد الكيماوي & $\varepsilon .0_{0}$ & RTr.. & مقاومة الآفات \\
\hline r.r. & $T r \cdot . T V$ & ثمن المبيدات & $1 \leqslant . r \leqslant$ & vol... & الحصناد و الجني \\
\hline $0 . \wedge T$ & $r .0 .$. & مصاريف نثرية & $r .94$ & T.O.TV & نقل المحصول \\
\hline $7 \varepsilon .9$ & rroo.rr & اجمالي التكاليف المتغيرة & $0 . \wedge \Gamma$ & $r .0 .$. & مصاريف نثرية \\
\hline$r 0.91$ & $1 \wedge \Lambda . .$. & الايجار & ro.91 & $1 \wedge \wedge . .$. & الايجار \\
\hline $1 \cdots, \cdots$ & OrTO.rT & المجموع الكلى للتكاليف & $1 \cdots \cdot$ & Orro.rr & المجمو ع الكلى للتكاليف \\
\hline
\end{tabular}

الصصدر: جمعت وحسبث من بيانات وزارة الزراعة واستصلاح الأراضي، قطاع الشئون الاقتصادية، الإدارة المركزية للاقتصاد الزراعي، نشرة الاقتصاد الزراعي، أعداد مختلفة.

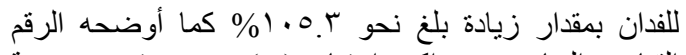

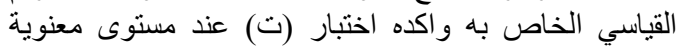

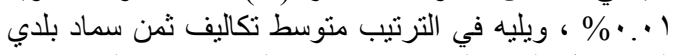

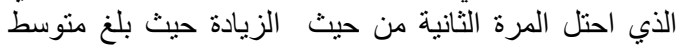

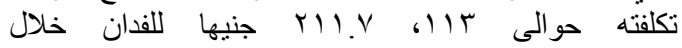

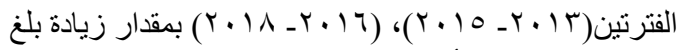

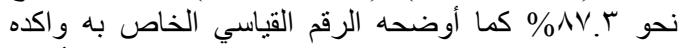

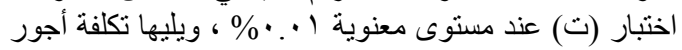

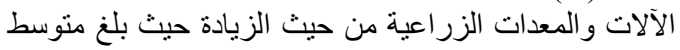

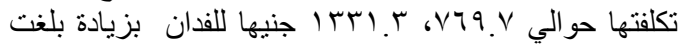

ب. أثر تغير سعر الصرف على بنود تكاليف إنتاج فدان القمح

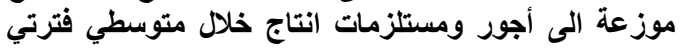

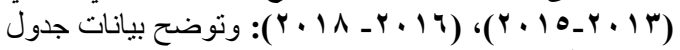

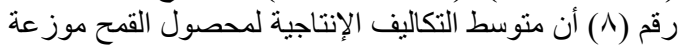

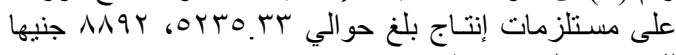

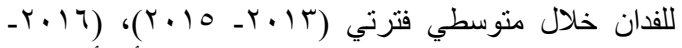

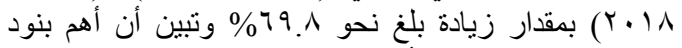

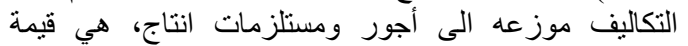

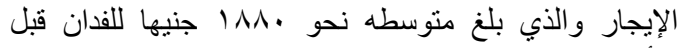
التأثير بتغيرات سعر الصرف وبلغت حوالي . بحىب جنية 


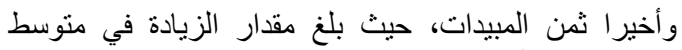

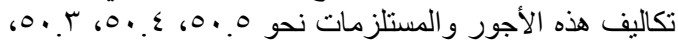

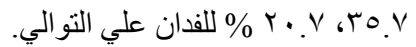

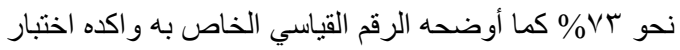

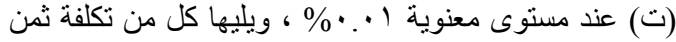

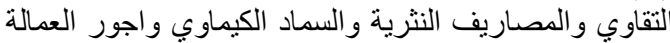

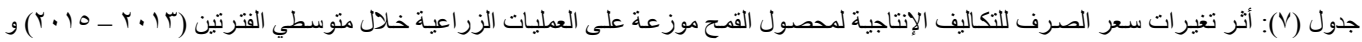

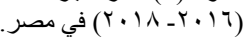

\begin{tabular}{|c|c|c|c|c|c|c|c|c|c|c|c|}
\hline \multirow{2}{*}{ اختبرار تار البيانات وفقا } & \multirow{2}{*}{ سعر الصرف فير } & \multirow{2}{*}{ للمتغير التياسي } & \multicolumn{4}{|c|}{ بعد تغير ات سعر صرف الجنيه المصري } & \multicolumn{4}{|c|}{ قبل تغير ات سعر صرف الجنيه المصري } & \multirow[b]{2}{*}{ العمليات الزراعية } \\
\hline & & & المتوسط الثاني & $r \cdot 11$ & $r+1 \mathrm{~V}$ & $r .17$ & | المتوسط الأول & $r .10$ & $r \cdot 1 \leq$ & T.IT & \\
\hline$* * V . V V$ & + & $1 \leq V . Y$ & rq9 & $\sum 7 V$ & $\varepsilon .0$ & rTo & TVI & Y97 & TVT & $T \leq \varepsilon$ & تحضير الأرض \\
\hline$* * 0, V V$ & + & $1 \leq 0.7$ & $0 \mathrm{ov} \cdot \mathrm{r}$ & 79. & $07 \mathrm{~V}$ & $\leq 0 \leq$ & rqI.V & 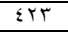 & r97 & rot & 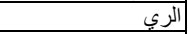 \\
\hline$* * \xi .1 Y$ & + & 101.7 & I.人r.r & $1 \leq \cdot r$ & 1.10 & 入. & $V \backslash \leq$ & $V V \cdot$ & $\mathrm{VI} \cdot$ & TIY & التسميد \\
\hline$* r . \leqslant r$ & + & IVT.9 & $19 V . V$ & $r 70$ & t.r & $1 \% 7$ & $11 \% . \mathrm{V}$ & 110 & 117 & $11 \cdot$ & الخدمة الزراعية \\
\hline *r.r & + & $1 \times 7.1$ & qEV.r & $118 \leq$ & АTr & 1.0 & Vol & VqY & VTr & 791 & الحصـاد أو الجنــى \\
\hline$* * 7 . \vee$ & + & $r \cdot 1.9$ & $\varepsilon r q . Y$ & 071 & $\varepsilon r$ & 190 & Y.O.Y & ro. & T. & 170 & نقــل المحصـــول \\
\hline$* * \varepsilon .0 Y$ & + & $10 \cdot .5$ & $\varepsilon 0 \wedge . V$ & $0 \wedge 1$ & $\varepsilon \varepsilon$ & ro. & $r .0$ & rTI & \begin{tabular}{|l|}
$r$ \\
\end{tabular} & rVA & مصـاريف أخـرى \\
\hline$* * \leqslant . \leqslant 7$ & + & 10. & $0 . \mathrm{Tr}$ & $7 \leq \leq 9$ & $\sum \vee 9 \lambda$ & $r \wedge \leqslant 9$ & rroo.r & rys. & \begin{tabular}{|l|l|}
$r+R \mid$ \\
\end{tabular} & $r .00$ & جمالي تكاليف العمليات \\
\hline
\end{tabular}

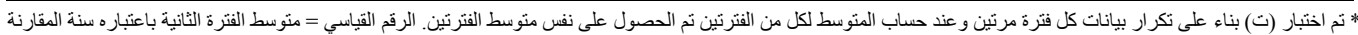

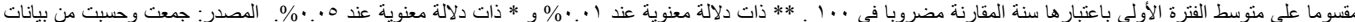

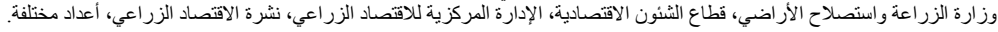

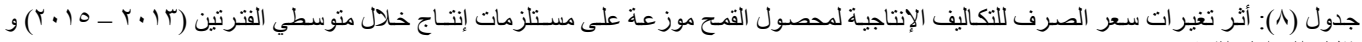

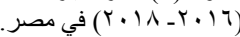

\begin{tabular}{|c|c|c|c|c|c|c|c|c|c|c|c|}
\hline \multirow{2}{*}{ لتكرار البيانات } & \multirow{2}{*}{ سعر الصنرف أثرات } & \multirow{2}{*}{ اللمتغيرات القياسي } & \multicolumn{4}{|c|}{ بعد تغيرات سعر صرف الجنيه المصري } & \multicolumn{4}{|c|}{ قبل تغير ات سعر صرف الجنيه المصري } & \multirow[b]{2}{*}{ العمليات الزراعية } \\
\hline & & & المنو سط الثاني & $r .11$ & $r . \mid \mathrm{V}$ & r.17 & المتوسط الأول & r. 10 & $r+1 \leq$ & $r .1 r$ & \\
\hline 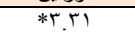 & + & Tro. & $T V \leqslant 0 . V$ & rYOY & 1094 & 1494 & TYAт.r & Tros & $\mid$ Irrq & 1187 & |أجور عمالة \\
\hline 1.01 & - & $\because$ & $\because$ & $\cdot$ & . & 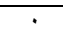 & r.r & . & $\cdot$ & 1. & |خدمسـة حيو انبة \\
\hline$* * 0.9 \varepsilon$ & + & IVT. & $14 \pi 1 . r$ & IVTY & $1 r \leqslant 9$ & $1 \cdots 1$ & V79.V & 190 & VTr & $7 V \mathrm{~V}$ & أجور الآلات \\
\hline$* Y . \wedge 9$ & + & 10.0 & sro. & $7 \ldots$ & ro. & rro & TAY.T & $r \cdot 1$ & $r 10$ & Yथा & اثمـن تقـــاوى \\
\hline$* 4.19$ & + & IAY.Y & rII.Y & YTY & YOV & 111 & $11 \%$ & 117 & $11 \%$ & 11. & ثمن سماد بلدي \\
\hline$* * 0 . \wedge 1$ & + & 10.4 & VIई. & NTו & VON & oor & $\leqslant \vee O_{0}$. & $01 \%$ & $\sum 79$ & 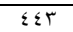 & إثمن سماد كيماوي \\
\hline$* \% .70$ & + & $T r \cdot . \mathrm{V}$ & $1 \leqslant 0 . y$ & 187 & 101 & 11. & $T+. Y$ & $\pi$ & TrY & $1 \ldots$ & ثئمـن ميبــات \\
\hline$* *$ ** Or & + & 10.5 & $\varepsilon 0 \Lambda . V$ & 011 & «६. & ro. & $r .0^{\prime}$ & TrI & $r .4$ & $r V A$ & مصـاريف أخـر \\
\hline$* * 11,19$ & + & $r .0 . r$ & rАт.. & हाAY & $\varepsilon 194$ & Tr.O & $1 \wedge \wedge .$. & $19 \mathrm{AV}$ & $19 \ldots$ & IVor & لإيجــار \\
\hline$* * Y, Y \uparrow$ & + & 179.1 & A१৭Y. & 1.741 & १११। & $V .0 \leqslant$ & orro.r & $07 Y V$ & orv & $\varepsilon \wedge \cdot \wedge$ & جمـالى تكاليف \\
\hline
\end{tabular}

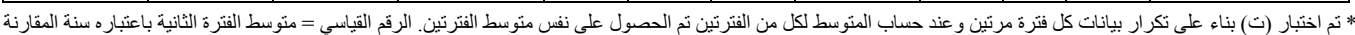

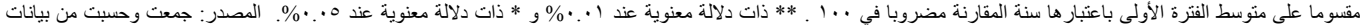

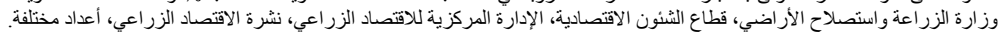

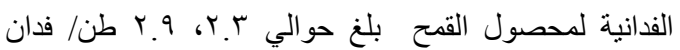

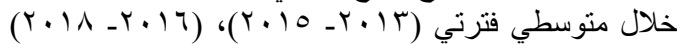

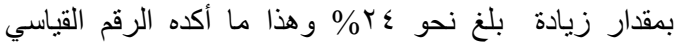

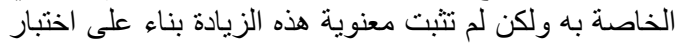

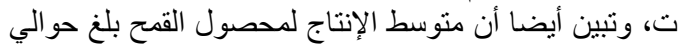

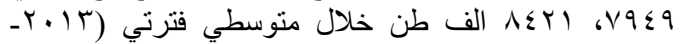
9. 0

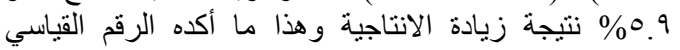

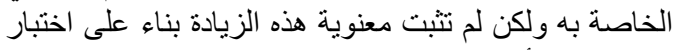

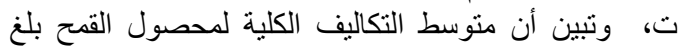

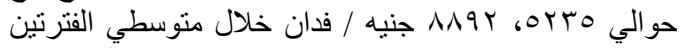

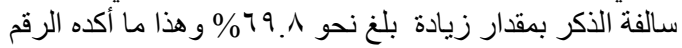

سادسا: أثر تغيرات سعر الصرف على بعض المؤشرات

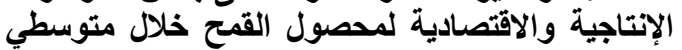
فترتي (r)

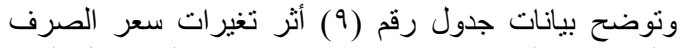

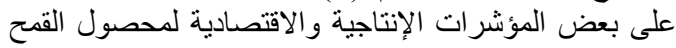

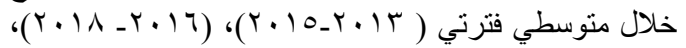

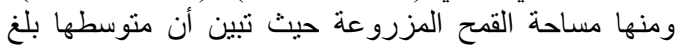

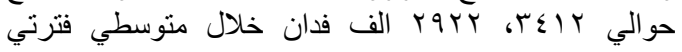
(Y)

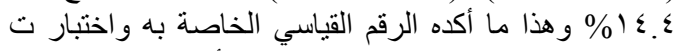
بمستوى معنوية عند ه . . \% الرثم ، كما تبين أن منوسط الإنتاجية 
القمح بينما لم تثبت معنوية النموذج المقدر لمتغير الإنتاجية

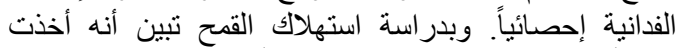

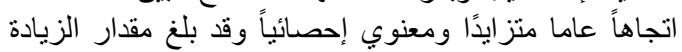

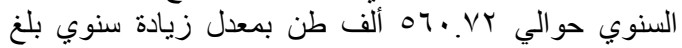

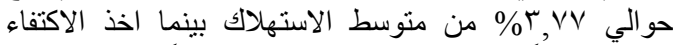

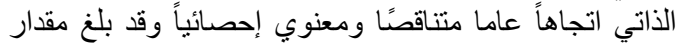

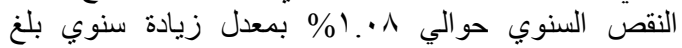

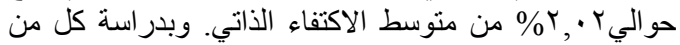

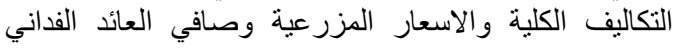

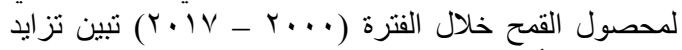

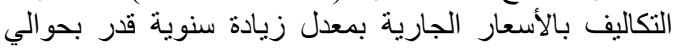

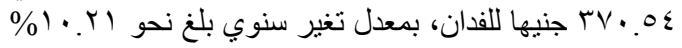

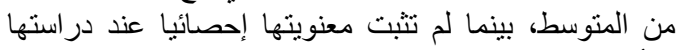

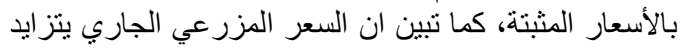

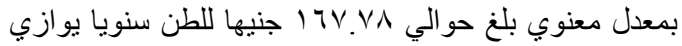

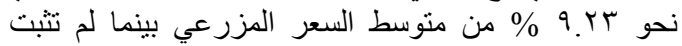

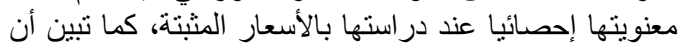

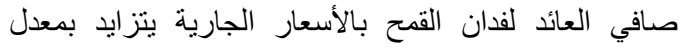

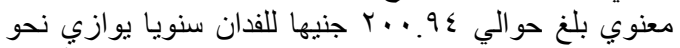

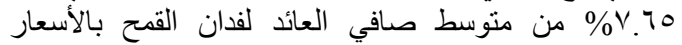

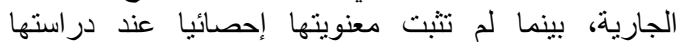

بالأسعار المثبتة.
القياسي الخاصة به واختبار ت بمستوى معنوية عند ا ل.. "

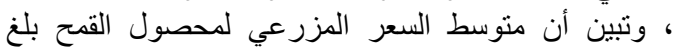

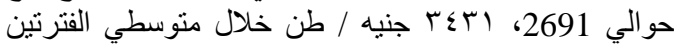

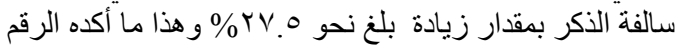

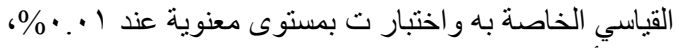

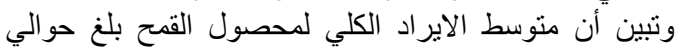

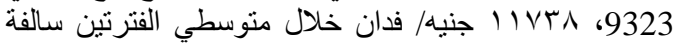

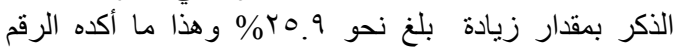

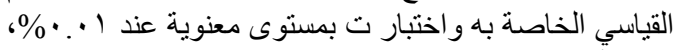

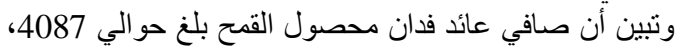

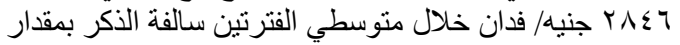

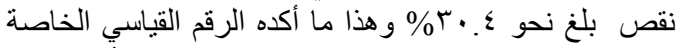

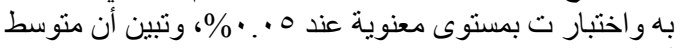

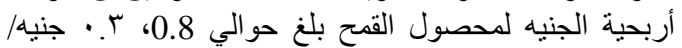

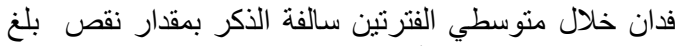

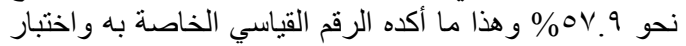

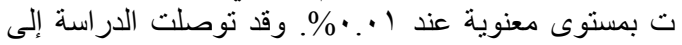

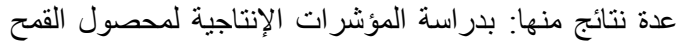

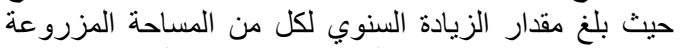

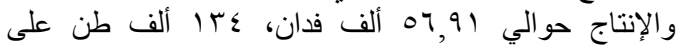

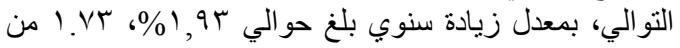

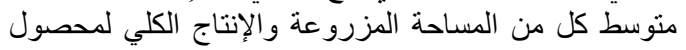

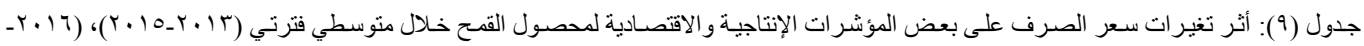
( $($ r. $) 1$

\begin{tabular}{|c|c|c|c|c|c|c|c|c|c|c|c|}
\hline \multirow{2}{*}{ اختبار تشر ار البيانات } & \multirow{2}{*}{ أثر تغير ات } & \multirow{2}{*}{ للمتغير ات اتسي } & \multicolumn{4}{|c|}{ بعد تغير ات سعر صرف الجنيه المصري } & \multicolumn{4}{|c|}{ قبل تغير ات سعر صرف الجنبه المصري } & \multirow[b]{2}{*}{ العمليات الزر اعية اعية } \\
\hline & & & 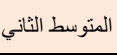 & r. 11 & $\mathrm{r} .1 \mathrm{~V}$ & $r .17$ & المنوسط الأول & $r+10$ & $r \cdot 1 \leq$ & $r+1 T$ & \\
\hline Y.AY* & - & 10.7 & T9YI.V & $r \leq q \cdot . r$ & rarI.V & \begin{tabular}{|l|l} 
reor.t \\
\end{tabular} & $r \leqslant ा t r$ & $r \leqslant 7 \wedge .9$ & $\begin{array}{ll}\text { rTar. } \\
\end{array}$ & rTVV.q & |المساحة المزرو عة \\
\hline. $\mathrm{r \Lambda}$ & + & 1.0 .9 & $\Lambda \leqslant Y ! .1$ & $v \leqslant 99.7$ & $\wedge \leq Y 1.1$ & $9 r \leq r .0$ & V9 & $97 \cdot V . V$ & ৭rV৭.^ & $\leqslant 97 . . Y$ & 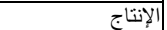 \\
\hline$* * Y . Y 7$ & + & 179.1 & १८१५. & $1.7 \pi 1 .$. & 1991.. & $V .0 \leqslant$. & OTrO. & OIYV.P. & orvi. & $\varepsilon \wedge \cdot \Lambda_{.} \cdot$ & |التكاليف الكلية \\
\hline$* * \varepsilon, Y \wedge$ & + & TrV.O & $01 \leq . V$ & $07 \leqslant$. & $07 \leqslant$. & $\leqslant 17$. & $\varepsilon \cdot r . y$ & $\Sigma 1 \%$. & $\Sigma 11$. & rAv. & |السعر المزرعي \\
\hline$* r . \wedge 0$ & - & 79.7 & $Y \wedge \leqslant 7 . r$ & $r I \leq r$. & rᄉrక. & YOVT.P & $\varepsilon \cdot \lambda V, r$ & rqs!. & $\varepsilon \cdot \leqslant V_{.} \cdot$ & $\varepsilon r V \leqslant .+$ & صافي العائد \\
\hline **YY.I & - & $\varepsilon Y .1$ & $\cdot r$ & $\cdot r$ & $\cdot \varepsilon$ & $\because \xi$ &.$\wedge$ & $\because V$ & $\because \wedge$ & .9 & أربحية الجنيه \\
\hline
\end{tabular}

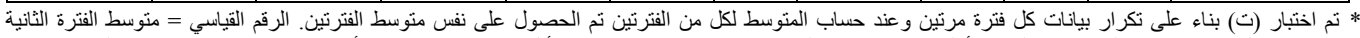

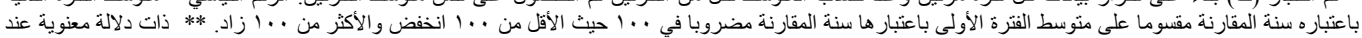

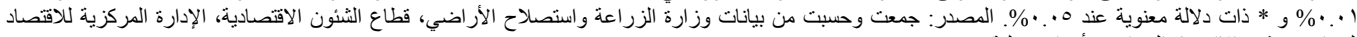
الزر اعي، نشرة الاقتصاد الزراعي، أعداد مختلفة.

سنوية متأثراً بتغيرات سعر صرف الجنيه المصري قدر

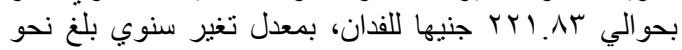

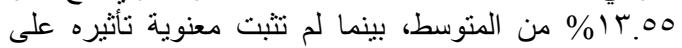

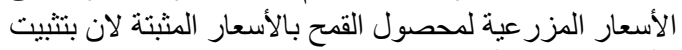

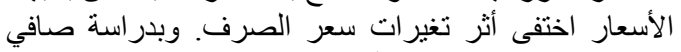

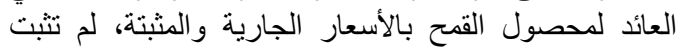

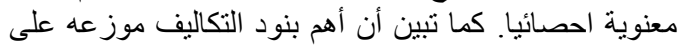

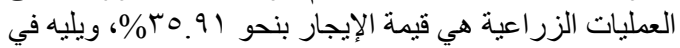

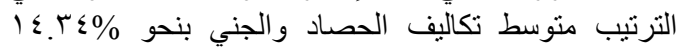

وبدراسة أثر تغيرات سعر الصرف على كل من التكاليف

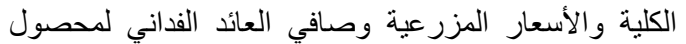

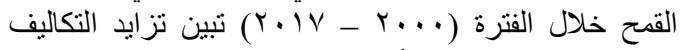

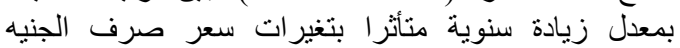

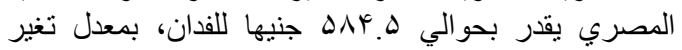

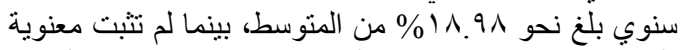

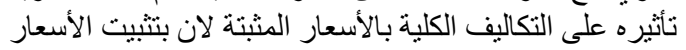

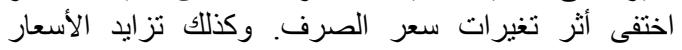

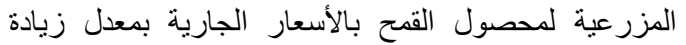


و المصاريف النثرية والسماد الكيماوي واجور العمالة وأخير الميال

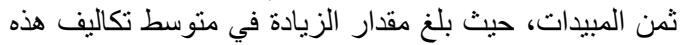

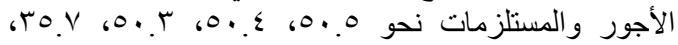

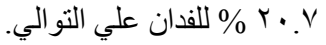

التوصيات

في ضوء النتائج فإن الدراسـة توصي باتخاذ العديد من التدابير

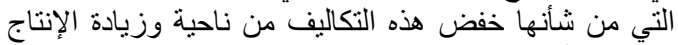

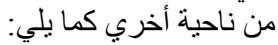

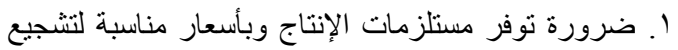

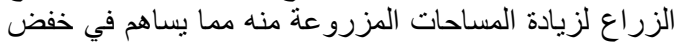

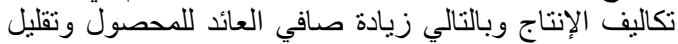

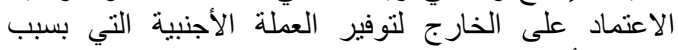
تغير اتها أدت الى زيادة التكاليف ومستلزمات الإنتاج وخاصة الإنة المستوردة.

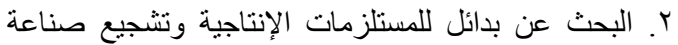

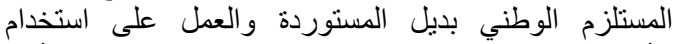

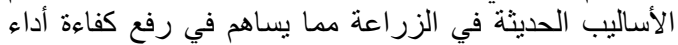

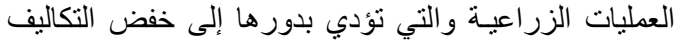
مما يوفر عائد مناسب للمز ارعين

\section{قائمة المراجع}

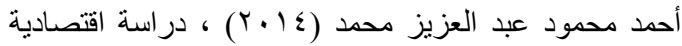

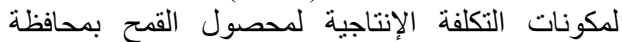

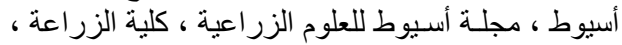

جامعة أسيوط ، مجلد (0 ـ ) ، العدد (0). البنك المركزي المصري ، جمهورية مصر العربية.

وزارة الزراعة واستصلاح الأراضي ، قطاع الثئون

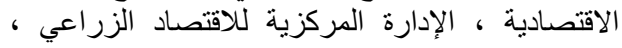

جمهورية مصر العربية ، نشرة الاقتصاد الزية الزراعي ، الزعية ، أعداد مختلفة.

الجهاز المركزي للتعبئة العامة والاحصاء ، جمهورية مصر

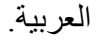

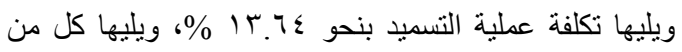

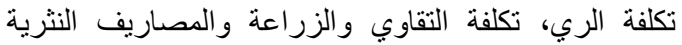

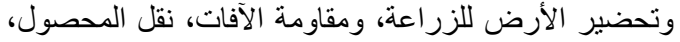

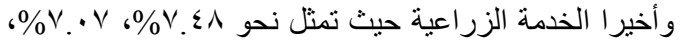
من \%

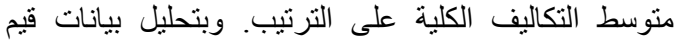

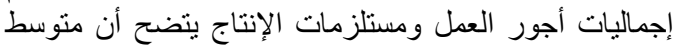

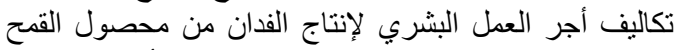

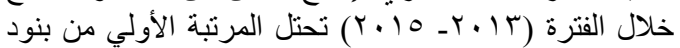

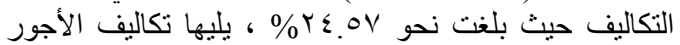

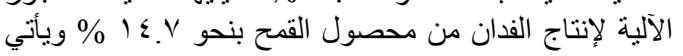

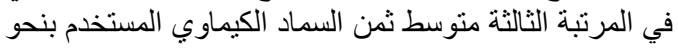

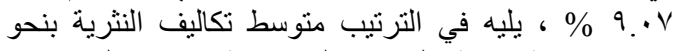

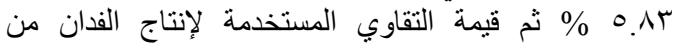

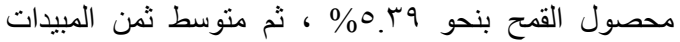

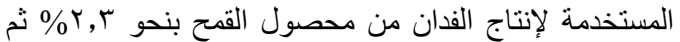

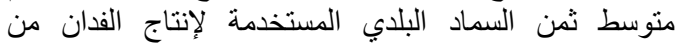

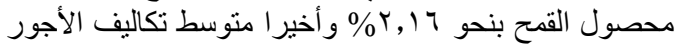

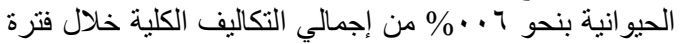

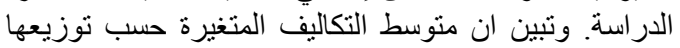

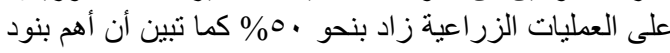

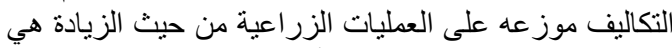

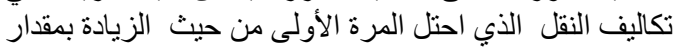

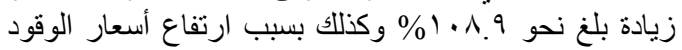

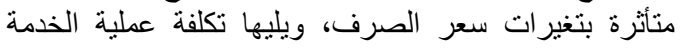

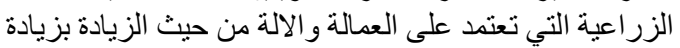

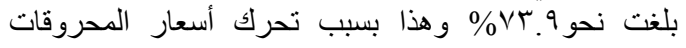

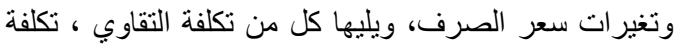

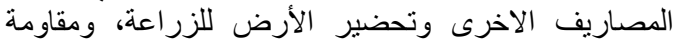

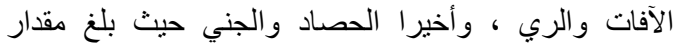

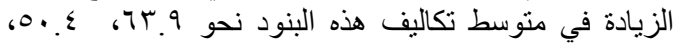

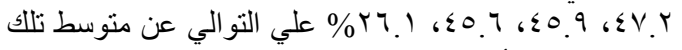

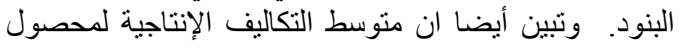

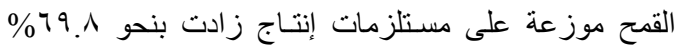

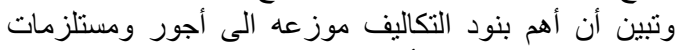

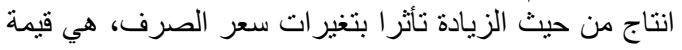

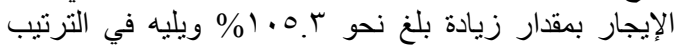

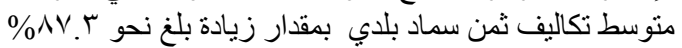

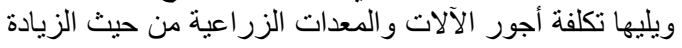

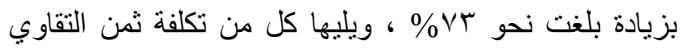

